\title{
Risque viral en AMP
}

\author{
R. LEVY \\ Laboratoire de Biologie de la Reproduction, CHU Nord, 42055 Saint Etienne
}

\section{RESUME}

De nombreux virus ont été détectés dans le sperme et sont par conséquent susceptibles d'être transmis au cours des actes d'Assistance Médicale à la Procréation (AMP). Le risque de contamination en AMP concerne la mère et l'enfant conçu, mais également les autres couples et le personnel technique manipulant les gamètes. Il pose un triple problème : de santé publique, éthique et juridique. Après un bref rappel des mesures de sécurité sanitaire en vigueur, ces différents points seront abordés au travers de 3 exemples = le cytomégalovirus (CMV), les virus des hépatites $B$ (VHB), C (VHC) et G (VHG), et le virus de l'immunodéficience humaine (VIH).

Mots clés : AMP, sperme, virus

\section{INTRODUCTION}

De nombreux virus ont été détectés dans le sperme : VIH [2], VHB [14], VHC [18], CMV [31], HSV [26], HPV [29]. La présence de ces virus dans l'éjaculat implique le risque de leur transmission en AMP : transmission à la mère et à l'enfant conçu, aux autres patientes traitées, au personnel technique du laboratoire lors de la manipulation des gamètes, de leur congélation, de leur conservation en paillettes dans l'azote liquide ou lors de leur décongélation [51].
Se pose alors un triple problème : de santé publique, éthique, juridique. En France, l'AMP participe pleinement au dépistage de certaines infections virales et la découverte d'une sérologie positive pour le VHB, le VHC ou le VIH lors du bilan préalable à la mise en route de l'AMP n'est pas exceptionnelle. Hors de France, des cas de transmission du VHB et du VIH en IAD ont été rapportés. Le pourcentage croissant de cas de contamination par le VHC en milieu hospitalier montre clairement le risque de transmission professionnelle et nosocomiale pour cet agent.

Si l'épidémiologie des agents viraux fait l'objet de nombreuses études, l'attitude pratique des centres d'AMP face à la prise en charge de patients infectés par le VHC, le VHB ou le VIH reste confuse. Se pose en effet le problème de répondre au désir d'enfant d'un couple dont l'un ou les deux membres présentent une maladie infectieuse parfois grave, dont l'évolution à court ou long terme est péjorative et dont le pronostic, malgré l'arrivée de nouveaux traitements, reste médiocre.

Entre 1994 et 1999, en l'absence de textes précis concernant l'AMP intra-couple, les directives de Santé Publique prônaient la prudence et l'abstention face à un couple infecté par le VHC ou le VIH, tout en laissant à chaque centre le libre arbitre. De nombreux centres ont résolu la question en refusant purement et simplement d'effectuer une AMP dans ces conditions. D'autres ont préconisé la création d'unités ultra - spécialisées dans le traitement de prélèvement biologiques infectés. 


\section{GUIDE DES BONNES PRATIQUES CLINIQUES ET BIOLOGIQUES}

La parution au Journal Officiel du 28 Février 1999 de l'Arrêté modifiant la loi de Bioéthique de Juillet 1994 reprend les principales définitions des mesures d'AMP, propose l'application de mesures universelles de précaution et propose une attitude pratique de prise en charge de patients infectés.

\section{Quelques définitions}

" L'AMP doit toujours répondre à une demande parentale, pour remédier à une infertilité dont le caractère pathologique a été médicalement constaté ou pour éviter la transmission à l'enfant d'une maladie d'une particulière gravité ".

Deux situations peuvent être ainsi envisagées. Dans le cas de couples infertiles nécessitant le recours à l'AMP, la prise en charge d'un homme infecté par un agent viral (VHC par exemple) pose le problème de transmettre lors de l'AMP le virus à une femme indemne, voire à l'enfant conçu. Tout autre est la situation de couples fertiles, séro-discordants pour le VIH, l'homme étant séropositif pour ce virus, et souhaitant avoir recours à l'AMP pour éviter tout risque de transmission à la future mère et à l'enfant. La prise en charge de ces couples dans un programme de don de sperme correspond en tout point à la définition de l'AMP.

Mesures "universelles" de précautions sanitaires

Dans le cadre des précautions " universelles " à respecter pour les laboratoires travaillant sur des prélèvements humains, définies par le CDC d'Atlanta (MMWR no 2 spécial, 1987), le personnel du laboratoire doit suivre les règles suivantes : port de gants pour toutes les manipulations de produits humains, à changer chaque fois que nécessaire et nécessairement entre deux patients; port de masque pendant les manipulations ; lavage des mains et de la peau immédiat en cas de projection ; prévention des piqûres et coupures (collecteurs, interdiction de reboucher les aiguilles)

Il est également important de rappeler que la vaccination du personnel du laboratoire contre l'hépatite $\mathrm{B}$ (la seule dont nous disposons actuellement) est obligatoire.

\section{L'AMP intra conjugale}

La loi de Bioéthique du 29 juillet 1994 a défini les grandes lignes des mesures de sécurité sanitaire en AMP avec dons de gamètes, mais il a fallu attendre la parution au J.O. du 28 février 1999 de l'Arrêté du 12 janvier 1999 relatif aux règles de bonnes pratiques cliniques et biologiques en assistance médicale à la procréation pour disposer de conduites à tenir claires et précises dans le cadre de l'AMP intra - couple.

Les tests de sécurité sanitaires sont à effectuer " avant la mise en œuvre d'une AMP intraconjugale pour éviter le risque de contamination de prélèvements d'autres couples, du personnel et de l'enfant à naître ". Cette recherche doit être réalisée avant la première tentative et, pour les autres tentatives, si le délai depuis la dernière détermination est supérieur à douze mois.

"Il est nécessaire de rechercher des marqueurs biologiques d'infection et, lorsque cela est techniquement possible, d'infectivité par VIH 1, VIH 2, les virus des hépatites $\mathrm{B}$ et $\mathrm{C}$ et la syphilis chez les deux membres du couple".

Le législateur a également prévu les modes de prise en charge des couples infectés et statué en fonction du virus en cause.

Dans le cadre d'une séropositivité au VIH, la prise en charge des couples est possible, mais uniquement dans le cadre d'un protocole de recherche clinique pluridisciplinaire relevant des prescriptions de la loi Huriet comprenant l'avis d'un CCPPRB et validé par la CNMBRDP.

Dans le cas du VHB, l'AMP est autorisée dans tous les cas. Si l'homme est porteur de l'antigène $\mathrm{HBs}$, il conviendra de vacciner la conjointe séronégative préalablement à toute AMP. Si la femme est porteuse de l'antigène HBs, l'AMP est possible, mais le couple doit être averti de la nécessité de réaliser la sérovaccination spécifique de l'enfant à la naissance.

En cas de séropositivité pour le VHC avec virémie de l'un ou l'autre ou des deux membres du couple vis-à-vis de l'hépatite $\mathrm{C}$, la prise en 
charge des couples est uniquement possible dans le cadre d'un protocole de recherche clinique pluridisciplinaire, relevant des prescriptions de la loi Huriet comprenant l'avis d'un CCPPRB et validé par la CNMBRDP.

Ces directives étaient indispensables ; elles sont claires, mais lourdes et restrictives puisque la prise en charge d'un patient virémique pour le VHC nécessite l'élaboration d'un protocole de recherche pluridisciplinaire avec passage devant le CCPPRB puis avis de la CNMBDRP. Ces mesures bloquent la prise en charge des couples et retardent d'autant l'AMP.

En revanche, la prise en charge des couples infectés par le VHB, sans nécessité de protocole de recherche, pose le problème du risque de transmission professionnel (tout le personnel est-il effectivement vacciné ?) et nosocomial (les autres couples en AMP sont - ils tous vaccinés ?). Dans les 2 cas, VHB et VHC, il semble souhaitable de disposer au moins d'une détection d'ADN ou ARN viraux avec quantification de la charge virale sérique.

\section{L'AMP avec don}

Des règles de sécurité sanitaire (673-5-13 du CSP) sont obligatoires pour le recueil et l'utilisation de gamètes humains provenant de dons en vue de la mise en œuvre d'AMP. Un certain nombre d'analyses de biologie médicale doit être pratiqué chez le donneur de gamètes avant le don, à la recherche des marqueurs biologiques d'infection et, lorsque cela est techniquement possible, d'infectivité :

\section{VIH 1, VIH 2, HTLV 1, HTLV 2, VH B VHC et syphilis}

Si les résultats sont positifs, les donneurs ou les donneuses de gamètes sont exclus. S'ils sont négatifs, un délai de six mois doit être respecté pendant lequel le sperme provenant du don ne peut pas être cédé, et les embryons issus d'ovocytes cédés ne peuvent pas être transférés. A l'issue de ce délai, les textes réglementaires prévoient que le bilan soit renouvelé pour les infections, à l'exception de HTLV 1 et HTLV 2. Il est cependant recommandé de les pratiquer. Le praticien est tenu de s'assurer que les résultats des analyses sont demeurés négatifs. Dans le cas où l'un ou plusieurs de ces résultats seraient devenus positifs, les gamètes ne pourraient être cédés ni les embryons transférés. En pratique, les analyses effectuées sont la recherche d'anticorps (sérologies). La détection des ARN ou ADN viraux dans le sérum ou, mieux encore, dans le prélèvement (don) lui-même par technique de biologie moléculaire n'est pas requise.

\section{Sérologie CMV (Ig M et IgG)}

Si le premier test est positif, en l'absence d'Ig $\mathrm{M}$, et après la période réglementaire de six mois, il est recommandé de n'utiliser les gamètes que pour les couples receveurs dont l'un au moins des membres est positif pour le CMV. La loi ne considère donc pas le cas particulier des couples " séro-discordants " où l'homme est séropositif pour le CMV et la femme séronégative, donc à risque potentiel de développer une primo - infection à CMV. En présence d'IgM, traduisant une infection récente, le don est récusé. Si le premier test est négatif, il doit être répété à l'issue de la période de six mois. Lorsque le deuxième test de CMV se positive, traduisant une infection récente, le don est récusé. Il est enfin recommandé de réserver les gamètes provenant de donneurs négatifs pour le CMV (moins de 50\% des donneurs) à des couples receveurs dont les deux membres sont eux-mêmes négatifs.

\section{Auto conservation}

L'auto conservation de sperme doit être systématiquement proposée aux patients avant toute thérapeutique ou intervention potentiellement stérilisante (chimiothérapie, radiothérapie, chirurgie...). Elle peut aussi être préconisée avant ou au cours d'une tentative d'AMP. Dans tous les cas, le bilan du patient comprendra en particulier la recherche des maladies infectieuses transmissibles : VIH 1 et VIH 2, syphilis, VH B et VHC.

\section{CAS PARTICULIERS}

\section{Le cytomégalovirus}

Il s'agit d'un virus ubiquitaire dont la prévalence varie en fonction de l'origine géographique, l'âge et la classe socio-économique [56]. La primo-infection à CMV chez la femme 
enceinte est la première cause d'infection virale congénitale et représente 0,2 à $2,2 \%$ des nouveaux nés soit 40000 naissances par an aux USA et 1500 naissances en France. Elle est responsable de la maladie des inclusions cytomégaliques qui donne lieu à un handicap sévère dans plus de $90 \%$ des cas.

Afin d'étudier les conséquences d'une transmission sexuelle du CMV liée au sperme, une micro - injection d'ADN CMV dans des ovocytes murins fécondés a été effectuée. De sévères et fréquentes anomalies du développement intra utérin, des retards de croissance et de graves anomalies neurologiques ont ainsi été décrits chez les foetus obtenus après micro -injection d'ADN CMV [7]. Cette étude soulève le risque de transmission en AMP du CMV, en particulier après ICSI. Cependant, la micro injection d'une quantité très importante d'ADN CMV purifié [5-20 molécules) directement au sein du pro noyau mâle ne peut être comparée à la faible quantité d'ADN CMV fixée à un spermatozoïde infecté micro - injecté dans un ovocyte humain.

Dans le but de quantifier le risque de transmission du CMV en AMP, nous avons étudié le risque de survenue d'une primo - infection à CMV en AMP intra-couple [31]. Les sérologies de 81 couples infertiles ont permis de retrouver une séroprévalence pour le CMV voisine de $60 \%$, mais ont surtout mis en évidence un groupe de patients à risque de primo - infection : il s'agit de couples où l'homme est séropositif (IgG) et la femme séronégative pour le CMV qui représente $13,5 \%$ des couples suivis. Dans le but de détecter les prélèvements infectés susceptibles de transmettre le CMV, nous avons recherché chez 130 patients (100 sujets séropositifs, 30 séronégatifs) la présence, dans le sperme, de l'ADN CMV (PCR) et d'un effet cytopathogène en culture cellulaire : $3 \%$ des spermes analysés provenant des sujets séropositifs pour le CMV étaient positifs en PCR et culture cellulaire. Aucun des spermes provenant des sujets séronégatifs ne présentait d'ADN CMV. Enfin, la détection d'ADN CMV dans le culot cellulaire après lavage simple, mais surtout après centrifugation en gradient de densité 3 couches s'est révélée faiblement positive, indiquant la persistance d'une faible quantité d'ADN CMV dans la fraction utilisée en AMP.

Par le biais d'études rétrospectives, deux équipes ont plus spécifiquement étudié le problème du CMV en don de sperme. Les CECOS de Toulouse et Montpellier ont étudié la présence du CMV dans 178 paillettes provenant de 97 donneurs $(50 \%$ séropositifs pour le CMV) et ont retrouvé 5 paillettes $(2,8 \%)$ de 2 donneurs (2\%) positives par culture cellulaire. Par amplification génique, 10 paillettes $(5,6 \%)$ provenant de 5 donneurs $(5,1 \%)$ ont été retrouvées positives par l'un ou l'autre des 2 centres (35]. Les auteurs soulignaient le risque potentiel de transmission du CMV par des paillettes en IAD, et discutaient de l'opportunité de remplacer les sérologies requises par les textes de loi par des techniques de recherche de l'ADN directement sur le sperme, plus performantes, mais également sujettes à discordance. En matière de recherche d'ADN CMV dans le sperme, comme pour toute technique de biologie moléculaire, il est absolument nécessaire de définir les conditions d'une PCR standardisée (amorces, réglages du thermocycleur). De plus, le sperme est un milieu biologique particulier car renfermant en quantité variable des enzymes pouvant interférer avec l'enzyme intervenant dans le processus d'amplification génique (Taq polymerase) et communément appelées " inhibiteurs de la Taq polymerase". La présence de ces inhibiteurs doit être impérativement détectée car ils peuvent être à l'origine de réactions faussement négatives. Une autre étude rétrospective effectuée en Belgique permet de comparer deux politiques en matière de don de sperme [32, 33]. L'une consiste à ne pas prendre en compte la sérologie CMV : cette attitude mène à un risque de contamination en IAD estimé à $21 \%$, ce risque étant fixé à $17 \%$ en transfusion sanguine. L'autre attitude prend en compte la sérologie CMV pour apparier donneur et receveuse : elle est possible puisque le centre note $50 \%$ de donneurs séronégatifs et $50 \%$ des femmes receveuses séronégatives pour le CMV et offre un risque nul puisque aucune séroconversion n'a été notée chez les receveuses. Enfin, l'absence de risque de transmission du CMV après transfert d'embryons obtenus dans un programme de don d'ovocytes a été récemment décrit [59]. 


\section{Les hépatites virales}

\section{Hépatite $B$ (VHB)}

Les facteurs de risque du VHB sont bien connus : toxicomanie, transfusion sanguine, dons d'organe avant 1988, tatouage, hémodialyse, rapports sexuels non protégés, personnel de santé non vacciné. Le VHB a été détecté dans la plupart des liquides biologiques : sang, salive, sperme, sécrétions vaginales, liquide pleural, LCR, ascite, lait maternel, liquide amniotique. Sa transmission était essentiellement sanguine ; d'autres modes de transmission sont maintenant prépondérants : sexuelle (présence du virus et de l'AgHBs dans le sperme), familiale (transmission par la salive), et materno - fotale (voie hématogène, per - partum, et allaitement). On doit à Berry la description du 1er cas de transmission du VHB en IAD [8]. Le VHB a été mis en évidence dans les leucocytes du liquide séminal [14] et détecté dans les cellules de la lignée germinale [21].

La transmission per - conceptionnelle du VHB a pu être clairement observée lors de la mise en évidence d'un milieu contaminé par le VHB [47] ayant provoqué 79 hépatites aiguës. 12 femmes infectées ont donné naissance à 16 enfants totalement indemnes grâce à une politique de sérovaccination prophylactique systématique à la naissance. L'infection des gamètes, non démontrée pour les ovocytes, est fortement suspectée pour les spermatozoïdes.

L'hépatite $\mathrm{B}$ n'est pas une contre indication à la grossesse : il n'y a aucune majoration de la gravité de l'hépatite $B$ (aiguë, chronique) en cas de grossesse. En revanche, les conséquences d'une infection par le VHB sur l'enfant ne sont pas négligeables. S'il n'existe pas d'effet tératogène, ni de retard de croissance intra utérin, ni d'anomalies du développement lié au VHB, une prématurité accrue, des maladies congénitales et infections post natales persistantes ont été décrites. L'enfant infecté par le VHB présente également un risque de mort prématurée par complications tardives (cirrhose, hépato carcinome).

En matière de dépistage préalable à l'AMP, les marqueurs biologiques à rechercher sont, au minimum, l'antigène HBs et les anticorps antiHBs. L'intérêt d'une recherche et quantifica- tion de l'ADN VHB dans le sérum doit être souligné. En effet, lorsque la charge virale est $<5$ $\mathrm{pg} / \mathrm{ml}$, le risque de transmission mère - enfant est nul. En revanche, si la charge virale est > $1000 \mathrm{pg} / \mathrm{ml}$, la contamination de l'enfant en cours de grossesse est certaine, avec, de plus, la possibilité d'un échec de la sérovaccination à la naissance.

En fonction de ce bilan, plusieurs situations peuvent se présenter. Si la femme est porteuse du virus (connue ou dépistée), le risque de transmissibilité à l'enfant est fonction de l'ADN VHB (PCR). Si la charge virale est nulle, après vaccination du conjoint et information du couple, la FIV pourra être effectuée. Si la charge virale est très élevée, la FIV sera retardée et effectuée après diminution ou annulation de la charge virale. Dans les 2 cas, une sérovaccination prophylactique systématique de l'enfant sera réalisée à la naissance. Si l'homme est atteint, le problème est différent. Après vaccination de la partenaire, la FIV est légalement autorisée. Cependant, la présence du virus dans le sperme est clairement établie, et fonction de la quantité d'ADN VHB sérique. La charge virale sérique, où, mieux encore, la recherche de l'ADN du VHB dans le sperme, permettra d'estimer le risque de manipuler des gamètes infectés.

\section{Hépatite C (VHC)}

Le problème du risque de transmission virale lié au VHC se pose en des termes totalement différents. Actuellement, aucune prévention n'est possible (pas de vaccin) et aucun traitement ne procure une efficacité complète et définitive. Le traitement de référence demeure l'interféron alpha à la dose de 3 millions d'unités 3 fois par semaine par voie sous cutanée pendant 12 mois. La réponse à ce traitement permet de distinguer 3 groupes de patients : les répondeurs dans 10 à $45 \%$ des cas, les rechuteurs (dès l'arrêt du traitement) dans près de $50 \%$ des cas et les non répondeurs. L'association de la ribavirine à l'interféron alpha est intéressante à considérer chez les patients naïs comme chez les rechuteurs : ses effets secondaires en contre-indiquent l'utilisation dans un processus d'AMP [28].

Les recommandations en cas d'infection à VHC 
varient singulièrement selon les centres et selon la spécialité des intervenants $[9 ; 16 ; 17$; 45]. Aucun consensus n'est clairement établi. Certains centres refusent d'effectuer une AMP dans ces conditions. D'autres préconisent la création d'unités spécialisées dans le traitement de prélèvements biologiques infectés. Le récent décret d'application impose l'établissement d'un protocole de recherche clinique pluridisciplinaire avec avis aux 2 commissions CCPRB et CNMBDRP.

En France, l'hépatite C est un important problème de santé publique et l'on estime le nombre de personnes infectées à environ 600 000, dont seulement 15000 cas diagnostiqués, parmi lesquels plus de 500000 sont porteurs du virus [48]. La voie de transmission du VHC est avant tout sanguine [19]. Le mode de contamination du VHC est maintenant identifié dans plus $70 \%$ des cas : $37 \%$ sont des malades transfusés (avant 1990) ou des hémophiles (avant 1987), $23 \%$ ont un antécédent de toxicomanie IV. $3 \%$ des contaminations seraient liées à une exposition professionnelle (profession de santé) [25]. La transmission par voie sexuelle a été décrite, mais fortement controversée : observée dans moins de $5 \%$ des cas, elle semble soit liée à des rapports traumatiques, ou pendant la période menstruelle, soit à des lésions génitales, et s'apparenterait aux accidents d'exposition au sang (AES) [12]. La transmission nosocomiale est retrouvée dans un nombre croissant de cas et concernerait jusqu'à $15 \%$ des contaminations par le VHC (biopsies endoscopiques, hémodialyse, soins médicaux et chirurgicaux invasifs) [1]. Des investigations d'épidémie à VHC survenue dans un hôpital du Sud de la France suggèrent clairement une transmission du VHC liée à l'utilisation d'un auto-piqueur pour l'auto mesure de la glycémie [15]. Le risque de transmission mère enfant a donné lieu à de nombreuses controverses [44, 58]. Ce risque concerne avant tout les mères co - infectées par le VIH. En dehors de ce contexte, ce risque est nul si la mère à une charge virale nulle, ou faible $(<3 \%)$ si la mère présente une infection active (virémie $\mathrm{C}$ supérieure à un million de copies / ml) [49].

La grossesse chez les patientes infectées par le
VHC n'induit aucune aggravation de l'hépatopathie et il n'existe pas de fotopathie connue induite par le VHC : il n'y a donc pas de contreindication à la grossesse chez les patientes infectées par le VHC.

L'évolution de la maladie suit un schéma simple : $80 \%$ des infections évoluent vers la chronicité ; chez les patients atteints d'une hépatite chronique $\mathrm{C}$, environ $20 \%$ vont évoluer vers la cirrhose en 15 ans et la progression vers le cancer hépato cellulaire est estimée à 5\% [52]. L'histoire de la maladie est toutefois aggravée par 3 facteurs : l'alcool, la co - infection par le VIH et l'âge (tardif) de la contamination [50].

Une étude récente quantifiait la population de couples infertiles concernés par le VHC : $2 \%$ environ des couples suivis en FIV [60]. La prise en charge en FIV d'un couple infertile présentant une infection active à VHC relève clairement d'un protocole de recherche pluridisciplinaire type loi Huriet depuis le 28-02-1999. De façon schématique, 3 situations peuvent être envisagées.

La réalisation d'une AMP en cas d'infection non active (ARN viral non détectable dans le sérum) ne pose aucun problème, si l'état du patient est satisfaisant (ALAT, PBH, échographie).

En cas d'infection active, la recherche et la quantification de l'ARN viral dans le sérum est un élément important. Chez la femme présentant une virémie $\mathrm{C}$ élevée, le risque de transmission verticale mère - enfant est faible, mais ne peut être négligé. De plus, si la présence d'ARN VHC dans les liquides folliculaires n'a jamais été publiée, elle a été retrouvée par certaines équipes, essentiellement dans les liquides hémorragiques. La réalisation d'une ponction folliculaire écho guidée sous anesthésie générale ou locale pose alors un réel problème de stérilisation au bloc opératoire, et représente un risque de manipulation de prélèvements infectés, ajoutant les risques de transmission sur un mode professionnel et nosocomial.

La réalisation d'une AMP pour un patient virémique pose un problème différent. De nombreux travaux détectent la présence $[18,27$, 
$34,42]$ ou l'absence $[20,23,57]$ du VHC ou de son ARN dans le sperme. L'étude de Semprini est la première qui stipule la recherche systématique d'inhibiteurs de la Taq polymerase dans le sperme et ses fractions : elle se veut rassurante puisque les auteurs concluent à l'absence d'excrétion du VHC dans le sperme [55]. En 1996, pourtant, l'ARN du VHC était détecté dans les paillettes d'un donneur infecté par le VHC et les auteurs démontraient la possibilité de réduire ou d'éliminer le risque infectieux lié au sperme par la réalisation d'un gradient de densité, permettant d'envisager la réalisation d'une AMP chez les hommes virémiques [39]. Ainsi, dans ce cas, la recherche systématique du génome du VHC dans le sperme, et surtout dans la fraction du sperme utilisée pour l'AMP, éventuellement associée à une congélation de sperme analysé, permettrait, si elle est négative, d'éliminer tout risque de manipulation d'un prélèvement infectieux le jour de la FIV.

\section{Hépatite $G$ (VHG)}

Le pouvoir pathogène du virus de l'hépatite $G$ (VHG) est encore mal connu : le VHG n'est incriminé que dans d'exceptionnelles hépatites fulminantes et peut-être dans certaines aplasies médullaires. La détection de l'ARN du VHG repose sur la PCR et depuis peu sur la recherche d'anticorps anti - enveloppe (anti -E2) qui apparaissent après négativation de la virémie. Le VHG est très souvent associé au virus de l'hépatite $C$ : dans l'étude de Semprini, environ un tiers des patients infectés par le VHC ont un ARN du VHG détectable dans le sang, et, pour six d'entre eux, la présence du VHG est confirmée dans le liquide séminal [55]. La transmission sexuelle (et en AMP) du VHG semble donc possible. Là encore, la controverse existe : Hollingsworth a en effet récemment affirmé l'absence d'ARN VHG détectable dans le plasma séminal [22].

\section{VIH}

La détection du VIH dans le sperme reste un sujet fortement débattu, et soulève encore de nombreuses questions.

Comment se fixe le VIH sur le spermatozoïde?

La recherche d'une molécule analogue au CD4, présente au niveau du spermatozoïde et pouvant servir de récepteur au VIH a abouti à l'identification d'un glycolipide spermatique, localisé sur la pièce intermédiaire et capable d'interagir avec la glycoprotéine d'enveloppe gp120 du VIH-1[13].

\section{Où?}

Une récente étude italienne analysant des testicules de patients décédés à différents stades de la maladie retrouve la présence d'ADN pro viral HIV-1 dans les noyaux des cellules germinales à tous les stades de leur différenciation et conclut à la possibilité de la production et de l'excrétion de spermatozoïdes infectés [41]. Les patients séropositifs mais asymptomatiques conservaient une spermatogenèse normale alors que chez les sujets présentant un SIDA déclaré, on observait une fréquente hypoplasie avec arrêt de la spermatogenèse. Cette inquiétante étude confirme les résultats de Nuovo qui, dès 1994, démontrait la présence du VIH dans les spermatogonies, les spermatocytes et, à un degré moindre, les spermatides [43]. Cependant, les deux études ont été effectuées sur des testicules provenant de sujets décédés et il semble difficile de pouvoir extrapoler à partir de ces seules données.

Dans le sperme, la détection et la localisation du VIH sont sujettes à de nombreuses controverses. Le VIH a été détecté par culture dans le sperme dès 1984, puis par PCR en 1994. Mermin en a retrouvé par PCR dans le liquide séminal et les cellules nucléées, mais jamais dans les spermatozoïdes [40], bien que Baccetti soit parvenu à visualiser des particules infectieuses sur mais surtout dans les spermatozoïdes de patients $[2,3,4,5]$. Quayle retrouve la présence du pro virus VIH dans $75 \%$ des spermes : en isolant les populations cellulaires par billes magnétiques, les auteurs notent que seules les cellules cibles du VIH, les lymphocytes $\mathrm{T}$ (75\% des échantillons) et les macrophages $(38 \%)$ sont infectés, jamais les spermatozoïdes mobiles [46]. L'étude marseillaise de Bréchard confirme l'absence du virus VIH dans les spermatozoïdes vivants et mobiles, et conclut à la présence inconstante du VIH dans le sperme, expliquant ainsi la transmission sur un mode occasionnel [10, 11]. Le risque faible, mais clairement non négligeable des rapports 
programmés (roulette russe) avec ou sans stimulation ovarienne simple, associé à l'absence totale de virus dans les spermatozoïdes mobiles sélectionnés après gradient de densité corroborée par de nombreuses études et en utilisant différentes méthodes (culture, co culture sur cellules humaines de sang de cordon, PCR $\mathrm{ADN}$ et $\mathrm{ARN}$ pro viral), a amené certaines équipes à répondre à la demande parentale des couples sérodiscordants pour le VIH où l'homme seul est atteint et à envisager la possibilité de pratiquer des actes d'AMP dans ce contexte particulier.

\section{AMP ET VIH}

\section{Questions techniques}

L'attitude des centres européens d'AMP en matière de sécurité sanitaire, et notamment du dépistage du VIH, est très disparate [30]. Lors d'une récente enquête, sur 70 centres anglais interrogés, seuls 23 effectuent une recherche d'anticorps anti-VIH, 24 tiennent compte du VHB et moins de $10 \mathrm{du}$ VHC [6].

Les premières IAC avec du sperme lavé ont été effectuées par Semprini dès 1992 [54]. De 1992 à 1997 , plus de 1000 cycles d'IAC ont été ainsi effectués et 250 enfants sont nés sans qu'aucun cas de contamination de la partenaire inséminée et de l'enfant ainsi conçu ait été noté [53]. L'équipe espagnole de Marina a récemment publié ses résultats en IAC IU [36] : 101 cycles d'IAC avec 37 enfants, sans aucun cas de contamination. La détection des ARN et ADN du VIH-1 par PCR sur la fraction des spermatozoïdes mobiles obtenue après gradient de densité Percoll 3 couches était effectuée en 5-7 heures, permettant d'effectuer l'insémination le jour même. Plus récemment encore, la même équipe a obtenu la première grossesse après ICSI [37]. Dans ce cas, la technique utilisée était identique, mais la fraction était mise en paillettes et l'ICSI effectuée à distance du prélèvement. Dans le même temps, en mars 1998, une équipe allemande décrivait le premier cas de contamination VIH-1 en IAD lié à l'utilisation de sperme frais provenant d'un sujet virémique, mais n'ayant pas encore d'anticorps sériques détectables, confirmant, s'il en était besoin, la nécessité d'une période de quarantaine de 6 mois pendant laquelle aucun prélèvement ne peut être utilisé [38].

\section{Questions éthiques}

Alors que les procédures de prise en charge en AMP des couples sérodiscordants se multiplient en Europe [30] et en France [24], il semble nécessaire de considérer l'aspect éthique et d'analyser la particularité que revêt le désir d'enfant légitime de ces couples. Peuton ainsi répondre à une demande parentale sans tenir compte de l'état actuel du patient, de l'évolutivité de l'infection et du pronostic à court ou long terme de la maladie ? Peut -on comme l'équipe de Marina effectuer une ICSI pour un couple dont le partenaire à un stade avancé de la maladie (C3 selon la classification du CDC) a présenté une toxoplasmose cérébrale et se maintient à $<200$ copies $/ \mathrm{ml}$ (et 75 $\mathrm{CD} 4 / \mathrm{mm} 3)$ sous thérapie lourde?

\section{Que proposer aux couples sérodiscor- dants?}

La première solution représente pour le couple un risque nul puisqu'elle fait appel au don de sperme : un nombre croissant de CECOS prennent désormais en charge les couples sérodiscordants qui se plient au mode de fonctionnement de ces centres : entretien avec un psychologue ou psychiatre, délai d'attente réglementaire, étude du dossier en réunion pluridisciplinaire comprenant le gynécologue, le biologiste, le psychologue assistés de l'infectiologue. D'autres couples préfèrent prendre le risque d'une AMP intra-couple. Après information claire et détaillée sur les méthodes employées pour réduire ou éliminer le VIH du sperme et leurs risques, les techniques classiques d'AMP (IAC, FIV ou ICSI) peuvent être envisagées après traitement du sperme et recherche de l'ARN et ADN pro viral du VIH par les techniques de biologie moléculaire.

\section{CONCLUSION}

La présence de virus dans le sperme implique le risque de leur transmission en AMP. La découverte de nouveaux agents infectieux, viraux ou autres, montre clairement que les précautions universelles recommandées doivent être mises en œuvre pour tous les prélèvements qui sont potentiellement dangereux. Par ailleurs, chaque virus présente des caractéristiques épidémiologiques et physiopathologiques particulières qui nécessitent une démarche spécifique. 
La prise en charge des patients infectés par l'un de ces agents dépend alors de données scientifiques (meilleure connaissance de l'épidémiologie et la physiopathologie, maîtrise assurée de la détection dans le sperme par les méthodes de biologie moléculaire), mais également de données éthiques avec la prise en compte par une équipe pluridisciplinaire du couple, de l'évolutivité de la maladie, de la place et de la valeur du désir d'enfant.

Enfin la disparité des conduites des centres européens en matière de sécurité sanitaire nous amène à souhaiter une homogénéité des mesures européennes, pas seulement en matière de dons de gamètes.

\section{RÉFÉRENCES}

1. AllaNDER T., GRUBER A., NAGHAVI M., et al. : Frequent patient-to-patient transmission of hepatitis $C$ virus in a haemotology ward. Lancet, 1995, 345 : 603-7.

2. BACCETTI B., BENEDETTO A. , BURRINI AG. : HIV particles detected in spermatozoa of patients with AIDS. J. Submicr. Cytol. Pathol., 1991, 23 : 339345.

3. BACCETTI B., BENEDETTO A. ,BURRINI AG. : HIV-1 particles in spermatozoa of patients with AIDS and their transfer into the oocytes. J. Cell Biol., 1994, 127 : 903-914.

4. BACCETTI B., BENEDETTO A., BURRINI AG. : Spermatozoa of patients with AIDS contain HIV particles. 1990, In Mélica, F. (ed.) AIDS and Human Reproduction. Karger, New York, pp. 47-54.

5. BACCETTI B., COLLODEL G., PIOMBONI P. : The debate on the presence of HIV-1 virus in human spermatozoa. In Fantini, J. and Sabatier, JM (eds), Perspective in Drug Discovery and Design. ESCOM Science Publishers B.V., Vol.5, pp.129-142.

6. BALET R., LOWER AM., WILSON C., ANDERSON J., GRUDZINSKAS JG. Attitudes towards routine human immunodeficiency virus (HIV) screening and fertility treatment in HIV positive patients - a UK survey. Hum. Reprod. 1998, 13 : 1085-7.

7. BASKAR J. F., FURNARI B., HUANG E. S. Demonstration of developmental anomalies in mouse fetuses by transfer of murine cytomegalovirus DNA injected eggs to surrogate mothers. J. Infect. Dis. $1993 ; 167: 1288$ - 1295.

8. BERRY WR., GOTTESFELD RL., ALTER HJ. Transmission of hepatitis $B$ virus by artificial insemination. JAMA, 1987, $257:$ 1079-81.

9. BOTTA - FRIDLUND D. : Risque de transmission des virus $\mathrm{B}$ et $\mathrm{C}$ en procréation médicalement assistée (PMA). Contracept. Fertil. Sex. 1994, 22, 298 - 301.
10. BRECHARD N., GALEA P., SILVY F., AMRAM M., CHERMANN JC. HIV virus detection in ejaculates collected at different times in seropositive patients. Contracept. Fertil. Sex. 1997, 25 : 725-9 (a).

11. BRECHARD N., GALEA P., SILVY F., AMRAM M., CHERMANN JC. Study of HIV localization in sperm. Contracept. Fertil. Sex. 1997, 25 : 389-91 (b).

12. BRESTERS D., MAUSER - BUNSCHOTEN EP., REESINK HW. : Sexual transmission of hepatitis C virus. Lancet, 1993, 342 : 210 - 211.

13. BROGI A., PRESENTINI R., SOLAZZO D., PIOMBONI P., COSTANTINO-CECCARINI E. Interaction of human immunodeficiency virus type 1 envelope glycoprotein gp120 with a galactoglycerolipid associated with human sperm. AIDS Res Hum Retroviruses 1996, $12: 483-9$.

14. DAVISON F., ALEXANDER GJ., TROWBRIDGE R., FAGAN EA., WILLIAMS R. Detection of hepatitis B virus DNA in spermatozoa, urine, saliva and leucocytes of chronic HBs Ag carriers. A lack of relationship with serum markers of replication. J. Hepatol. 1987, 41, 37-44.

15. DESENCLOS JC., RAZES M., ROLIN B. et al. : Transmission nosocomiale du VHC documentée lors de l'investigation d'une épidémie hospitalière. $\mathrm{BEH}$, 1998, $7: 25-27$.

16. DUFFAUT M., VALLA D. : Procréation médicalement assistée et infection par le virus de l'hépatite C. Contracept. Fertil. Sex 1997, 25 : 534 - 537.

17. FEDERATION DES BLEFCO : Propositions d'attitudes en matière de dépistage des hépatites $\mathrm{B}$ et $\mathrm{C}$ préalables à la tentative d'AMP intra - couple. Contracept. Fertil. Sex 1997, .25, 313 - 324.

18. FIORE, RJ., POTENZA D., MONNO L. et al. : Detection of HCV RNA in serum and seminal fluid from HIV - 1 co - infected intravenous drug addicts. J. Med. Virol. 1995, 46, 4, 364 - 7 .

19. FRERY-GOURIER C., MERLE V., GORIA R. et al. : Facteurs de risque de transmission de l'infection par le virus de l'hépatite C. Résultats d'une enquête cas témoins en population générale. Rev. Epidemiol. Sante Pub., 1997, S43, C0040.

20. FRIED MW., SHINDO M., FONG T., et al. : Absence of hepatitis $\mathrm{C}$ viral RNA from saliva and semen of patients with chronic hepatitis C. Gastroenterology, 1992, 102 : 1306 - 1308.

21. HADCHOUEL M., SCOTTO J., HURET JL., MOLINIE C., VILLA E., DEGOS F. BRECHOT C. Presence of HBV-DNA in spermatozoa : a possible vertical transmission of HBV via the germ line. J. Med. Virol., $1985,16: 61-66$.

22. HOLLINGSWORTH RC., JAMESON CL., MINTON JE. et al. : GBV-C/HGV coinfection in HIV-1 positive men : frequent detection of viral RNA in blood plasma but absence from seminal fluid plasma. J. Med. Virol. $1998,56,321-6$. 
23. HSU HH., WRIGHT TL., LUBA, D. et al. : Failure to detect hepatitis $\mathrm{C}$ virus genome in human secretions with the polymerase chain reaction. Hepatology,1991, $14: 763-767$.

24. JOUANNET P., DULIOUST E., KUNSTMANN JM et al. : Management of fertile and infertile HIV positive patients wanting to become parents. Proc. 16th congr. On fertil. Steril. 1998 (sous presse).

25. KIYOSAWA K., SODEYAMA T., TANAKA E. : Hepatitis $\mathbf{C}$ in hospital employees with needlestick injuries. Ann. Intern. Med.1991, $115: 367$ - 369.

26. KOTRONIAS D., KAPRANOS N. Detection of herpes simplex virus DNA in human spermatozoa by in situ hybridization technique. In Vivo 1998, 14 : 391-4.

27. KOTWAL, G. J., RUSTGI, V. K., BAROUDY, B. M. Detection of hepatitis $C$ virus specific antigens in semen from non A, non B hepatitis patients. Dig. Dis. Sci. 1992, $35: 641-644$.

28. LAI M., KAO J., YANG P. et al. : Long-term efficacy of ribavirin plus interferon alfa in the treatment of chronic hepatitis C. Gastroenterol., 1996, 111 : 1307-12.

29. LAI YM., LEE JF. , HUANG HY., SOON YK., YANG FP., PAO CC. The effect of human papillomavirus infection on sperm cell motility. Fertil. Steril. 1997, 67 : 1152-5.

30. LEVY R., GUERIN JF., NAJIOULLAH F. : Screening Infertile Couples for HIV Infection. Fertil. Steril. $1998,70: 785-786$.

31. LEVY R., NAJIOULLAH F., KEPPI B. et al. : Detection of cytomegalovirus in semen from a population of men seeking infertility evaluation. Fertil. Steril., 1997, 68, 820 - 826.

32. LIESNARD CA. : Screening of semen donors for infectious diseases. Hum. Reprod. 1998, 13 Suppl 2 : 12-24 (a).

33. LIESNARD CA., REVELARD P., ENGLERT Y. : Is matching between women and donors feasible to avoid cytomegalovirus infection in artificial insemination with donor semen? Hum. Reprod. 1198, 13 Suppl $2: 25-31(b)$

34. LIOU T., CHANG TT., YOUNG KC. et al. : Detection of HCV RNA in saliva, urine, seminal fluid and ascites. J. Med. Virol., 1992, 37, 197 - 202.

35. MANSAT A., MENGELLE C., CHALET M., et al. : Cytomegalovirus detection in cryopreserved semen samples collected for therapeutic donor insemination. Hum. Reprod., 1997, 12 : 1663-1666.

36. MARINA S., MARINA F., ALCOLEA R. et al. : Human immunodeficiency virus type 1 - serodiscordant couples can bear healthy children after undergoing intrauterine insemination. Fertil. Steril., 1998, $70: 35-39$

37. MARINA S., MARINA F., ALCOLEA R., NADAL J., EXPOSITO R., HUGUET J. : Pregnancy following intracytoplasmic sperm injection from an HIV-1 sero- positive man. Hum. Reprod., 1998, 13 : 3247-9.

38. MATZ B., KUPFER B., KO Y., WALGER P., VETTER Y., EBERLE J., GÜRTLER L. : HIV-1 infection by artificial insemination. Lancet, 1998, 351 : 728 .

39. McKEE TA., AVERY S., MAJID A., BRINSDEN PR. Risks for transmission of hepatitis $\mathrm{C}$ virus during artificial insemination. Fertil. Steril., 1996, 66 : 1613.

40. MERMIN JH., HOLODNIY M., KATZENSTEIN DA, MERIGAN TC. : Detection of human immunodeficiency virus DNA and RNA in semen by the polymerase chain reaction. J. Infect. Dis., 1991, 164 : 769-72.

41. MUCIACCIA B., UCCINI S., FILIPPINI A., ZIPARO E., PARAIRE F., BARONI CD., STEFANINI $M$. Presence and cellular distribution of HIV in the testes of seropositive subjects : an evaluation by in situ PCR hybridization. FASEB J. 1998, 12 : 151-63.

42. NUMATA N., OHORI H., HAYAKAWA Y., et al. : Demonstration of hepatitis $C$ virus genome in saliva and urine of patients with type $C$ hepatitis : usefulness of the single round polymerase chain reaction method for detection of the $\mathrm{HCV}$ genome. J. Med. Virol., 1993, 41: 120 - 128.

43. NUOVO GJ., BECKER A., SIMSIR M. et al. : HIV-1 nuclei acids localize to spermatogonia and their progeny. A study by polymerase chain reaction in situ hybridization. Am. J. Pathol., 1994, 144 : 1142-1148.

44. OHTO H., TERAZAWA S., SASAKI N. et al. : Transmission of hepatitis $\mathrm{C}$ virus from mothers to infants. New Engl J Med 1994, 330 : 744 - 750.

45. PAWLOTSKY J. M. : Assistance médicale à la procréation et hépatites virales : la position du virologue. Contracept. Fertil. Sex., 1997, 25, 530 - 533.

46. QUAYLE AJ, XU C., MAYER KH., ANDERSON DJ. : $T$ lymphocytes and macrophages but not motile spermatozoa are a significant source of human immunodeficiency virus in semen. J. Infect. Dis., 1997, 176 : 960-8.

47. QUINT WG., RIJNTJES J., HEITJINK RA. Hepatitis $B$ virus $B$ DNA by gene amplification in women accidently infected at In Vitro Fertilization (IVF) and their offspring. Abstract $\mathbf{n}^{\circ} 213$, International Symposium on viral hepatitis and liver disease (Houston, Texas), April 4-8, 1990.

48. ROUDOT - THORAVAL F et al. : Epidémiologie et morbidité du virus de l'hépatite $\mathrm{C}$ en France. Etude de 6664 patients atteints d'hépatite chronique. Bull. Epidémiol. Hebdo.,1996, 4 : 20 - 21.

49. ROUDOT - THORAVAL F., PAWLOTSKY, JM., THIERS V. et al. : Lack of transmission of hepatitis $C$ virus in human immunodeficiency virus seronegative women : a prospective study with hepatitis $\mathrm{C}$ virus RNA testing. Hepatology, 1993, 17 : 772-7.

50. ROUDOT - THORAVAL, F., BASTIE, A., PAWLOTSKY, J. M., DHUMEAUX, D. ET LE GROUPE D'ETUDE DE LA PREVALENCE ET DE LEPIDEMIOLO- 
GIE DES HEPATITES C : Prévalence, gravité et facteurs de risque des hépatites chroniques $\mathrm{C}$ en France: enquête nationale (résumé) Gastroenterol. Clin. Biol., 1994, $18: 1080$.

51. RUSSELL PH, LYARUU VH, MILLAR JD, CURRY MR., WATSON PF The potential transmission of infectious agents by semen packaging during storage for artificial insemination. Anim. Reprod. Sci. 1997, $47: 337-42$.

52. SAITO I., MIYAMURA T., OHBAYASHI A., et al. : (1990) Hepatitis $C$ virus infection is associated with the development of hepatocellular carcinoma. Proc. Natl. Acad. Sci. USA,1990, 87 : 6547 - 9.

53. SEMPRINI A. Reproductive counselling for HIV-discordant couples. Lancet, 1997, 349 : 1401-2.

54. SEMPRINI A., LEVY-SETTI P., BOZZO M., et al. : Insemination of HIV-negative women with processed semen of HIV-positive partners. Lancet, 1992, 340 : 1317-9.

55. SEMPRINI A., PERSICO T., THIERS V. et al. Absence of hepatitis $C$ virus and detection of hepatitis $G$ virus / GB virus C RNA sequences in the semen of infected men. J. Infect. Dis. 1998, $177: 848-54$.

56. STAGNO S., WHITLEY R. J. Herpesvirus infections of pregnancy. Part I: Cytomegalovirus and Epstein Barr virus infections. N. Engl. J. Med. 1985 ; 313, 1270 - 1274.

57. TERADA, S., KAWANISHI, K., KATAYAMA, K. : Minimal hepatitis $\mathrm{C}$ infectivity in semen. Annals Intern. Med., 1992, $117: 171$ - 172.

58. WEJSTAL, R., WIDELL, A., MANSSON, A., HERMODSSON, S., NORKRANS, G. Mother to infant transmission of hepatitis $\mathrm{C}$ virus. Ann. Intern. Med., $1992,117: 887$ - 890.

59. WITZ CA., DUAN Y., BURNS WN., ATHERTON SS., SCHENKEN RS. Is there a risk of cytomegalovirus transmission during in vitro fertilization with donated oocytes ? Fertil. Steril. 1999, 71 : 302-307.

60. ZORN, J. R., EPELBOIN, S. : Hépatite C et assistance médicale à la procréation intra conjugale. La Revue du Praticien Gynécologie et Obstétrique, 1997, 14, 24 -27 .

\author{
ABSTRACT \\ Transmission viral risk in assisted \\ reproductive technics (art)
}

R. LEVY

Detection of virus (VIH, CMV, HSV, HPV, $\mathrm{HCV}, \mathrm{HBV}$ ) in semen raises the issue of the risk of transmission of infection to the mother and the future baby, to other gametes and embryos in the incubator, and to technicians related to the manipulation of contaminated biological fluids in the standard IVF laboratory. The transmissibility of virus through medical techniques is a critical question in public health care. For each virus, it becomes essential to assess transmission risk in ART, especially in in vitro fertilization (IVF) with intracytoplasmic sperm injection (ICSI), to define the management of couples according to their viral status. Here, we studied different virus : CMV, HCV, HBV and HIV and proposed practical attitudes towards virus screening and management in infertility centers.

Keys words : ART, semen, virus 